\title{
Fatores de Risco para Infarto do Miocárdio no Brasil Estudo FRICAS
}

\author{
Marco Aurélio Dias da Silva, Amanda G. M. R. Sousa, Hernan Schargodsky
}

São Paulo, SP

\begin{abstract}
Objetivo-Determinar os fatores de risco para a ocorrência de infarto agudo do miocárdio (IAM) no Brasil.

Métodos - Estudo conduzido entre janeiro/94 e março/95, em 20 centros médicos no Brasil, constituído de casos, 299 pacientes com IAMe, controles, 292 indivíduos, identificados no mesmo centro que os casos, e admitidos com largo espectro de doenças agudas, não relacionadas a fatores de risco conhecidos ou suspeitos para IAM. Os dados foram colhidos por meio de um questionário estruturado, preenchido pelo próprio paciente. Os efeitos das variáveis pesquisadas sobre a ocorrência de IAMforam estudadas em abordagens univariadas, considerando-se significativo $p \leq 0,05$.
\end{abstract}

Resultados - Os fatores relacionados ao risco de IAM foram, para os casos e controles, respectivamente: hipercolesterolemia: $210,93 \pm 46,74 \mathrm{mg} / \mathrm{dl}$ e $185,71 \pm 45,45 \mathrm{mg} / \mathrm{dl}$ $(p=0,000)$; tabagismo: $41,69 \%$ e 27,20\% $(p=0,000)$; $h i-$ pertensão arterial: $52,35 \%$ e 20,88\% $(p=0,000)$; diabetes mellitus: $19,70 \%$ e 9,93\% $(p=0,001)$; história familiar: $p o-$ sitiva no pai dos indivíduos em 42,14 e 33,22\% $(p=0,025)$ e na mãe em 42,14\% e 30,82\% (p=0,007); situação socioeconômica: $88,99 \%$ e $60,20 \%$ proprietários de casa própria $(p=0,002)$ e 44,45\% e 33,21\% de automóvel $(p=0,010)$; atividade fisica: $56,83 \%$ e 48,28\% haviam mantido o hábito de caminhar no ano que antecedeu à entrada no estudo ( $p=0,029)$; hábitos alimentares: 38,79 e 28,42\% consumiam habitualmente embutidos ( $p=0,013)$. A média do peso corporal foi de $72,50 \pm 26,89 \mathrm{~kg}$ e $69 \pm 12,26 \mathrm{~kg}(p=0,0271) e$ a altura média de $166,56 \pm 7,81 \mathrm{~cm}$ e $166,66 \pm 8,47 \mathrm{~cm}$.

Conclusão - O estudo confirmou a importância da hipercolesterolemia, hipertensão arterial sistêmica, diabetes, sobrepeso e história familiar positiva, como fatores de risco para ocorrência de IAM. Houve relação direta e significativa entre a ocorrência de IAM e a condição socioeconômica.

Palvras-chave: infarto agudo do miocárdio, fatores de risco coronário, estudo caso-controle

\section{Risk Factors for Acute Myocardial Infarction in Brazil (FRICAS Study)}

Purpose - To determine risk factors related to acute myocardial infarction (AMI) in Brazil.

Methods - Five hundred ninety one patients were selected in 20 medical centers in Brazil, between January/ 94 and March/95. Two-hundred-ninety-nine patients were cases, and 292 controls with a variety of acute diseases not related to the suspected or known risk factors for acute myocardial infarction. All data were collected through a structured questionnaire filled by the patients. The evaluation of the variables was done through univariate analysis with a significance level of $5 \%$.

Results - The factors related to acute myocardial infarction were respectively to cases and controls: Hypercholesterolemia $-210.93 \pm 46.74 \mathrm{mg} / \mathrm{dl}$ and $185.71 \pm 45.45 \mathrm{mg} / \mathrm{dl}$, $(p=0.000)$; smoking $-41.69 \%$ and $27.20 \%(p=0.000)$; hypertension $-52.35 \%$ and $20.88 \%(p=0.000)$; diabetes $19.70 \%$ and $9.93 \%(p=0.001)$; family history - related to the patient's father in $42.14 \%$ and $33.22 \%(p=0.025)$ and to the mother in $42.14 \%$ and $30.82 \%(p=0.007)$; socioeconomic level $-88.99 \%$ and $60.20 \%$ owned house $(p=0.002)$; $44.45 \%$ and $33.21 \%$ owned car $(p=0.010)$; physical activity $-56.83 \%$ and $48.28 \%$ had the habit of walking in the year prior to study entry $(p=0.029)$; diet $-38.79 \%$ and $28.42 \%$ consumed canned food ( $p=0.013)$. The mean weight was $72.50 \pm 26.89 \mathrm{~kg}$ and $69 \pm 12.26 \mathrm{~kg}(p=0.0271)$. The mean height was $166.56 \pm 8.81 \mathrm{~cm}$ and $166.66 \pm 8.47 \mathrm{~cm}$.

Conclusion - This study confirmed the importance of hypercholesterolemia, hypertension, diabetes mellitus, overweight and family history as risk factors for acute myocardial infarction. There was a significant relation between socioeconomic level and the prevalence of acute myocardial infarction.

Key-words: acute myocardial infarction, coronary risk factors, case-control study

Arq Bras Cardiol, volume $71\left(n^{0}\right.$ 5), 667-675, 1998

Instituto Dante Pazzanese de Cardiologia

Correspondência: Marco Aurélio Dias da Silva - Rua Adalivia de Toledo, 182 05683-000 - São Paulo, SP

Recebido para publicação em 12/5/98

Aceito em 9/9/98
As doenças cardiovasculares (DCV) são as principais causas de morte e, também, de morbidade e incapacitação entre os países ocidentais desenvolvidos ${ }^{1}$. No âmbito das DCVs, a aterosclerose, tanto a das artérias coronárias como 
a cerebral, constitue-se, nesses países, em sério problema de saúde pública, assumindo contornos de verdadeira epidemia $^{2}$. No Brasil não é diferente: excluídos os óbitos de causas não definidas, as DCVs representaram, em 1988, 50\% do total de óbitos femininos e $40 \%$ dos óbitos masculinos, entre os com mais de 30 anos de idade ${ }^{3}$. O primeiro estudo longitudinal e prospectivo, sobre a epidemiologia das DCVs, envolvendo grande número de indivíduos, foi levado a efeito na cidade de Framinghan, nos Estados Unidos ${ }^{4,5}$. Os resultados desses estudo permitiram identificar um grupo de fatores que predispunham a um maior risco do desenvolvimento da aterosclerose, entre os quais despontavam, como mais importantes, a faixa etária, o sexo masculino, a hereditariedade, a hipercolesterolemia, a hipertensão arterial sistêmica (HAS) e o diabetes mellitus (DM). A partir da identificação desses fatores, iniciou-se persistente trabalho visando sua eliminação ou atenuação, de tal sorte que, na maior parte dos países ocidentais desenvolvidos, vem se percebendo, nas últimas décadas, reversão na tendência de mortalidade por doença arterial coronária (DAC) ${ }^{1}$.

Entre nós, há falta de estudo similar; as campanhas e a política de prevenção das DCVs, tanto as promovidas pelas agências oficiais, como pelas organizações não-governamentais, têm se baseado nos resultados do estudo de Framinghan e eleito o combate aos fatores de risco supracitados, como o seu alvo maior. A questão que se colocava e para a qual não se tinha resposta era: seriam aqueles fatores identificados, como sendo de risco para a população branca, majoritariamente anglo-saxã e rica, da Nova Inglaterra, válidos também para outros grupos populacionais, em especial para a América do Sul? Com a finalidade de responder a esta questão, identificar e quantificar os fatores que, nos diversos países do Continente, associam-se a um risco maior para o desenvolvimento de infarto do miocárdio, o Comitê de Epidemiologia da União das Sociedades de Cardiologia da América do Sul (USCAS) planejou, coordenou e elaborou um estudo multicêntrico sobre os Fatores de Risco para a Insuficiência Coronariana na América do Sul (FRICAS). No Brasil, a incorporação dos pacientes ao estudo iniciou-se em janeiro/94, concluindo-se em março/ 95. Objetivou-se, neste estudo, determinar a freqüência e importância com que os principais fatores de risco para DAC, apontados na literatura internacional, associam-se, em nosso meio, à ocorrência de infarto agudo do miocárdio (IAM).

\section{Métodos}

A população estudada constou de 591 indivíduos, distribuídos em dois grupos: os que sofreram IAM, diagnosticado no momento do recrutamento (grupo IAM), e aqueles não portadores de DCV (grupo controle). Não houve cálculo prévio quanto ao tamanho da amostra, embora o planejamento do estudo previsse a inclusão de 200 indivíduos no estudo, o que não foi alcançado. Os pacientes (casos e controles) foram agrupados por idade e sexo (tab. I) não havendo diferenças significativas entre os dois grupos, no que tange a estas variáveis. A participação no estu- do foi aberta, através de carta-convite, a todos os centros cardiológicos do país que dispunham de unidade coronária, conforme informação das coordenações regionais do FUNCOR. Participaram do estudo e recrutamento dos pacientes 20 centros, (tab. II), dos quais 13 situados no Estado de São Paulo; sendo que a cidade de Marília respondeu, isoladamente, por 169 pacientes (28,6\% do total). Os critérios para inclusão no grupo IAM foram: quadro clínico, caracterizado por precordialgia com duração superior a 30min; quadro laboratorial, baseado na elevação enzimática (CKMB) superior a, no mínimo, 50\% daquele considerado normal pelo método empregado e eletrocardiograma (ECG) mostrando novas ondas $Q$, segundo a classificação de Minnesota. No grupo controle, foi exigido, como critério de inclusão, que os pacientes fossem provenientes do mesmo centro do caso IAM com o qual emparelhar-se-ia e que, obrigatoriamente, fosse portador de, ao menos, uma das seguintes afecções: traumatológicas, abdominais agudas, oftalmológicas e otorrinolaringológicas agudas e, ainda, doenças de pele. Excluíram-se os portadores de doença cardiovascular, vascular periférica, vascular cerebral ou

\begin{tabular}{|lccc|}
\hline \multicolumn{4}{|c|}{ Tabela I - Características da amostra } \\
\hline & Masculino & Feminino \\
\hline & & 224 & 75 \\
Grupo IAM & 299 & 200 & 92 \\
Grupo controle & 292 & $\mathrm{p}=0,083$ & \\
Idade & & \\
IAM & 30 a 85 anos & $(58,68 \pm 12,07)$ anos \\
Controle & 27 a 85 anos & $(58,26 \pm 11,99)$ anos \\
\hline IAM- infarto agudo do miocárdio. \\
\hline
\end{tabular}

\begin{tabular}{|c|c|c|}
\hline \multicolumn{3}{|c|}{ Tabela II - Cidades participantes } \\
\hline Cidade & $\mathrm{N}^{\circ}$ de casos & $\%$ \\
\hline \multicolumn{3}{|l|}{ Estado de São Paulo } \\
\hline Andradina & 1 & 0,17 \\
\hline Araçatuba & 34 & 5,75 \\
\hline Araraquara & 1 & 0,17 \\
\hline Araras & 56 & 9,47 \\
\hline Duartina & 1 & 0,17 \\
\hline Leme & 7 & 1,18 \\
\hline Marília & 169 & 28,60 \\
\hline Presidente Prudente & 18 & 3,04 \\
\hline Santos & 36 & 6,09 \\
\hline São Carlos & 22 & 3,72 \\
\hline São José do Rio Preto & 16 & 2,70 \\
\hline São Paulo (Capital) & 46 & 7,78 \\
\hline Taiobeiras & 1 & 0,17 \\
\hline Total & 408 & 69,04 \\
\hline \multicolumn{3}{|l|}{ Outros Estados } \\
\hline Brasília (DF) & 20 & 3,38 \\
\hline Goiânia (GO) & 79 & 13,36 \\
\hline João Pessoa (BP) & 17 & 2,88 \\
\hline Manaus (AM) & 4 & 0,68 \\
\hline Porto Alegre (RS) & 10 & 1,69 \\
\hline São Luis (MA) & 20 & 3.38 \\
\hline Vitória/Vila Velha (ES) & 33 & 5,58 \\
\hline Total & 183 & 30,96 \\
\hline
\end{tabular}


doenças crônicas de qualquer tipo. O recrutamento dos pacientes, tanto os casos como os controles, deu-se a partir das unidades de emergência e/ou pronto-atendimento dos hospitais participantes. Os pacientes com suspeita de IAM eram obrigatoriamente submetidos a ECG e dosagem de enzimas séricas para confirmação diagnóstica. Adquirida a confimação, procedia-se o emparelhamento com um controle do mesmo sexo e idade aproximada (diferença de no máximo cinco anos). Para ambos preenchia-se um questionário estruturado, padronizado e idêntico e dosava-se o colesterol total, após $12 \mathrm{~h}$ de jejum. Através do questionário, foram analisadas as seguintes variáveis: hábitos de vida (tabagismo, atividade física. padrão de alimentação), antecedentes pessoais de HAS, DM, antecedentes de DAC na família, estado civil, nível sérico do colesterol total, atividade profissional (com relação à atividade física), situação socioeconômica, nível educacional e obesidade. A avaliação dos hábitos alimentares foi baseada na apresentação, ao paciente, dos alimentos pesquisados, cabendo-lhe assinalar quantas porções ou quantas vezes por semana os consumia. Não houve padronização ou especificação quanto à quantidade de alimentos em cada porção. Definiu-se como fumante aquele que consumia, regularmente, no mínimo, um cigarro ao dia, pelo menos durante um ano, e que havia fumado no ano anterior à inclusão no estudo; não-fumante aquele que nunca havia fumado ou, o havia feito há 10 anos e ex-fumante, o indivíduo que não fumara no ano anterior à inclusão no estudo, mas que o havia feito entre um e os 10 anos anteriores. No que tange à atividade física, pesquisou-se a prática de qualquer esporte físico e/ou hábito de caminhar, considerando-se como positiva, apenas, se praticada por no mínimo 45 min e três vezes por semana. Foram avaliadas tanto a atividade atual (se praticada no último ano) como no passado (qualquer época anterior). Com relação ao componente físico da atividade profissional, consideraram-se três aspectos: se aposentado ou em atividade, se a profissão era sedentária ou não, entendendo-se como não sedentária a atividade que obriga a caminhar mais da metade do tempo de trabalho e, ainda, se o tipo de trabalho implicava ou não em atividade manual pesada. Incluiu-se como hipertenso ou diabético, o paciente que recebia tratamento para essas condições ou referia esse diagnóstico emitido por médico. As informações concernentes à altura e peso basearam-se nas informações fornecidas pelo paciente, quando do ingresso, ou na aferição direta por parte do investigador, quando não havia segurança ou disponibilidade da informação. Os valores comparados foram as médias entre os dois grupos, \pm 0 desvio padrão, expressando-se a altura em centímetros e o peso em quilogramas. O indivíduo foi rotulado como tendo história familiar positiva para DAC quando relatava ter, pelo menos, um familiar direto (pais ou irmãos) que havia apresentado IAM. Para avaliação e estratificação da classe social levou-se em conta a posse de casa própria e/ou automóvel. O grau de instrução foi estratificado em três grupos: analfabeto, primário/médio e superior (completo). Analisaram-se também os hábitos alimentares, considerando-se quatro grupos de comparações: a) consumo de alimentos sabida-

\begin{tabular}{|c|c|c|}
\hline \multicolumn{3}{|c|}{ Tabela III - Peso Corporal } \\
\hline & Controle & Infartados \\
\hline Peso médio & $69+12,26 \mathrm{~kg}$ & $72 \pm 26,89 \mathrm{~kg}$ \\
\hline Altura média & $\begin{array}{c}166,66+8,47 \mathrm{~cm} \\
\mathrm{p}=0,0271\end{array}$ & $166,56 \pm 7,81 \mathrm{~cm}$ \\
\hline
\end{tabular}

\begin{tabular}{|lcc|}
\hline \multicolumn{3}{|c|}{ Tabela IV - Estado civil } \\
\hline & Infarto agudo do miocárdio & Controle \\
\hline Solteiro & $13(4,36 \%)$ & $18(6,16 \%)$ \\
Casado & $231(77,52 \%)$ & $204(69,86 \%)$ \\
Separado & $24(8,05 \%)$ & $29(9,93 \%)$ \\
Viúvo & $29(9,73 \%)$ & $13(7,00 \%)$ \\
\hline
\end{tabular}

mente ricos em gorduras de origem animal (embutidos, carne de porco, carne bovina, vísceras e frituras); b) consumo de queijo e leite integral comparado com o consumo de aves, peixes, frutas frescas e vegetais verdes; c) consumo de óleo de oliva em comparação a outros óleos vegetais; d) consumo de manteiga comparado ao de margarina; e) consumo de chá (inclusive mate) comparado ao de café. Para comparação dos níveis de colesterol total do sangue, consideraram-se as médias dos dois grupos \pm o desvio padrão, tendo os valores de concentração sérica sido expressos em miligramas por decilitro de sangue.

Análise estatística - Os efeitos das variáveis pesquisadas sobre a ocorrência de IAM foram estudadas em abordagens univariadas, aplicadas individualmente a cada variável de interesse. Variáveis discretas foram comparadas através do teste de diferença de proporções, enquanto que as variáveis contínuas foram comparadas através do teste de diferenças de médias (teste "t" de Student). Em ambos os casos adotou-se como valor crítico de significância o nível de $\mathrm{p} \leq 0,05$.

\section{Resultados}

A observação da idade e sexo dos casos de IAM, incluídos no estudo, (tab. I) mostra predominância do sexo masculino (2,98 homens para 1 mulher) e idade média de $58 \pm 12,07$ anos. Nas tabelas III e IV compararam-se, respectivamente, a relação/altura e o estado civil dos dois grupos. Observa-se, na tabela III, que a média de peso corporal dos infartados foi significativamente superior ao do grupo controle $(\mathrm{p}=0,0271)$, apesar da altura média ser semelhante, indicando maior prevalência de sobrepeso entre os casos de IAM. No que tange ao estado civil, não houve diferença significativa entre os dois grupos. As figuras 1 a 17 trazem os gráficos referentes às prevalências e/ou magnitude das demais variáveis analisadas e comparadas entre os dois grupos. Entre os que sofreram infarto, houve maior prevalência, estatisticamente significativa, tanto de pai $(\mathrm{p}=0,025)$ como de mãe ( $\mathrm{p}=0,007)$ com história de infarto (fig. 1). A associação com HAS (fig. 2) foi marcadamente superior entre os do grupo $\operatorname{IAM}(\mathrm{p}=0,000)$ como também o foi com o hábito de 


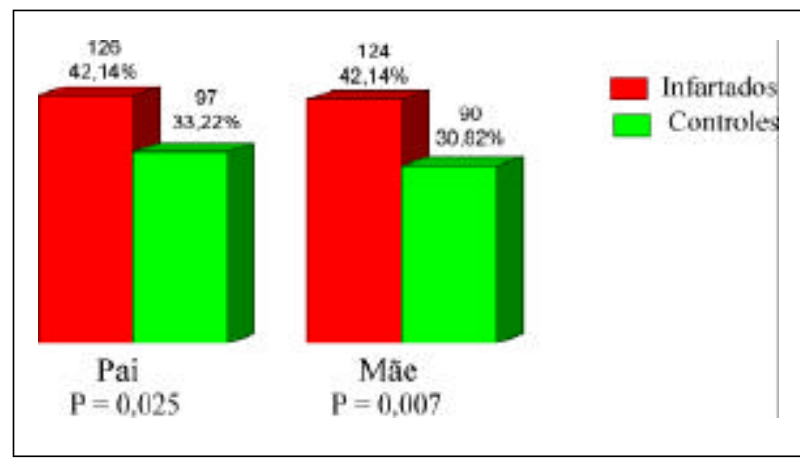

Fig. 1 - Antecedentes familiares positivos.

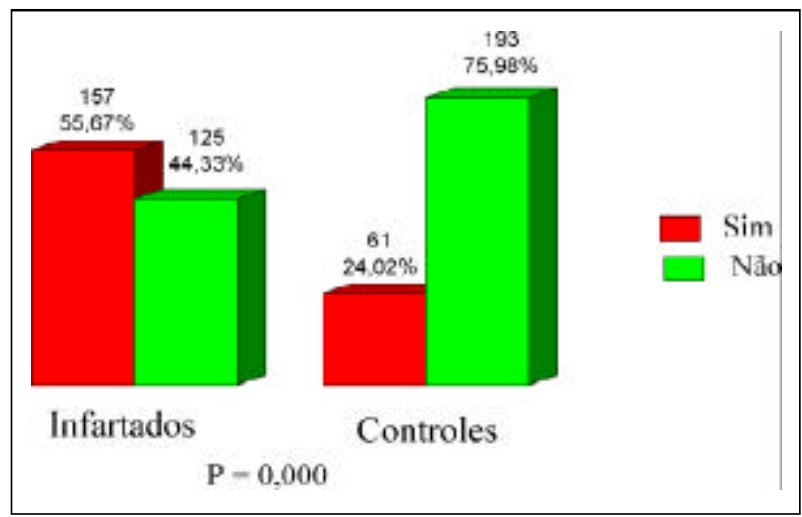

Fig. 2 - Associação com hipertensão arterial.

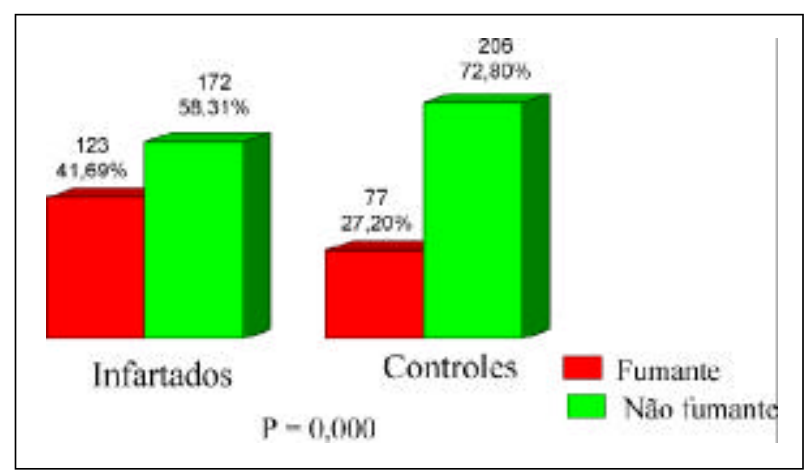

Fig. 3 - Associação com tabagismo I.

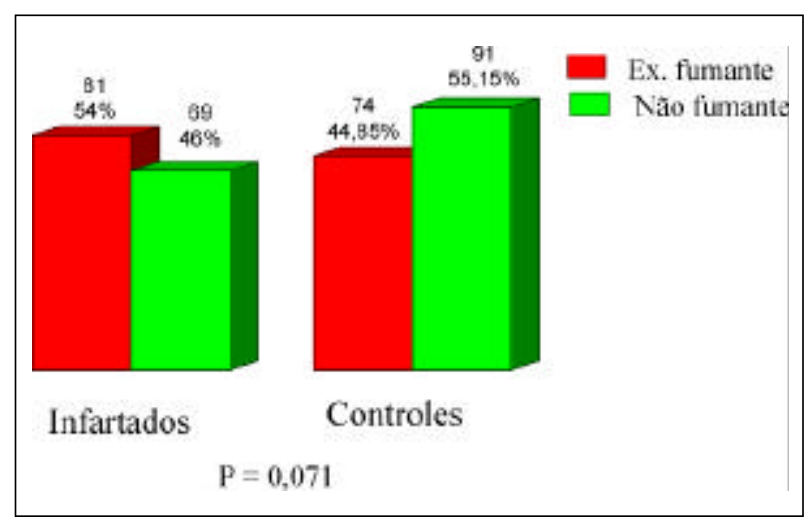

Fig. 4 - Associação com tabagismo II.

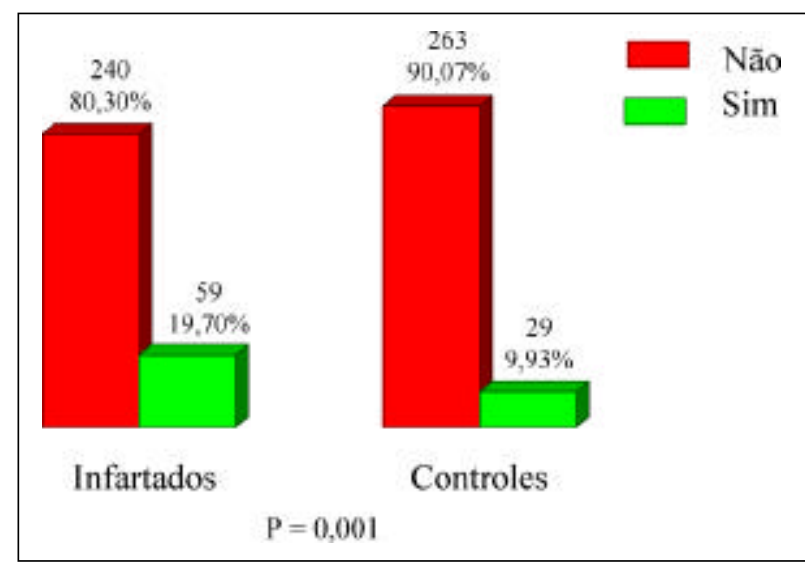

Fig. 5 - Associação com diabetes mellitus.

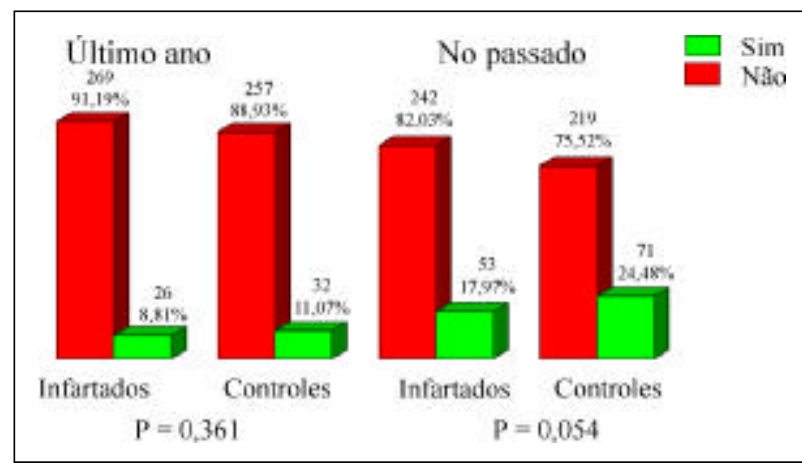

Fig. 6 - Prática de esportes. *Prática regular, sessões de no mínimo 45min.

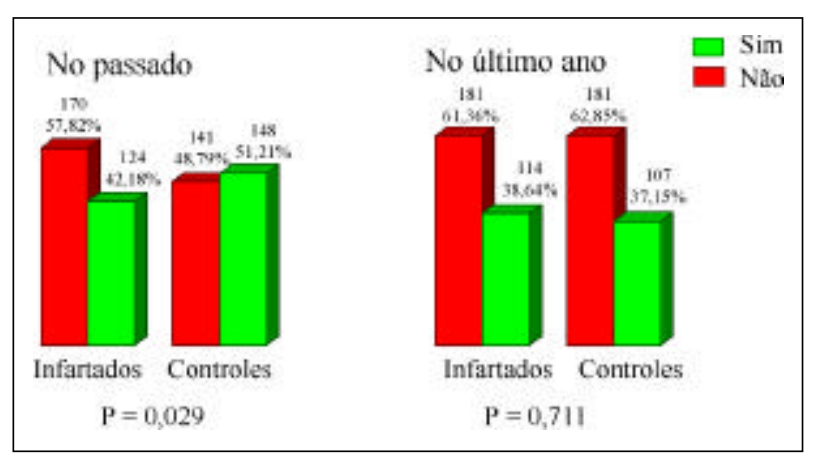

Fig. 7 - Hábito de caminhar. *Caminhar ou passear regularmente por mais de $45 \mathrm{~min}$

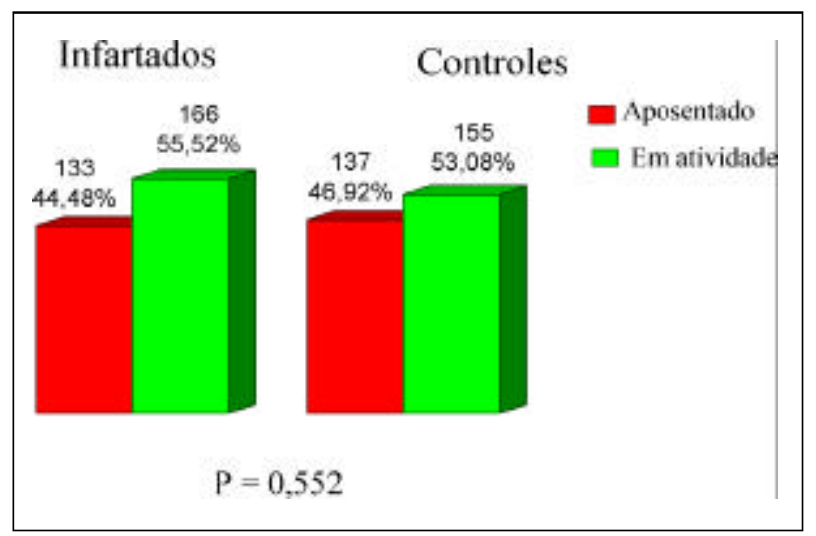

Fig. 8 - Atividade profissional I. 
fumar (fig. 3, p=0,000). Não houve, no entanto, diferença significativa entre os não fumantes e fumantes $(p=0,071)$, embora a ocorrência de IAM tenha sido mais freqüente entre os ex-fumantes (fig. 4). Observou-se também associação significativa $(\mathrm{p}=0,001)$ entre a presença de DMe IAM(fig. 5). Com referência à atividade física, pôde-se observar que a maioria dos pacientes de ambos os grupos não praticava ou praticava esportes (fig. 6), não se tendo verificado diferença significativa entre os dois grupos, tanto atual (último ano), como passada. Já com relação ao hábito de caminhar, (fig. 7) a prevalência de IAM foi significativamente menor entre os que cultivaram este hábito no passado ( $\mathrm{p}=0,029)$, não constatando-se diferença nos dois grupos entre os que o faziam noúltimo ano $(\mathrm{p}=0,711)$. Não houve, também, diferença significativa entre os que se mantinham em atividade profissional comparados aos aposentados (fig. 8, p=0,552), nem com relação ao tipo de atividade, se sedentária ou não(fig. 9), tanto atual $(p=0,648)$ como no passado $(p=0,601)$. O fato dotipo de trabalho exigir atividade manual pesada (fig. 10) não mostrou diferença significativa entre os dois grupos, tanto atual $(\mathrm{p}=0,223)$ como no passado $(\mathrm{p}=0,862)$. A prevalência do IAM foi significativamente maior entre os de melhor situação econômica (fig. 11), tanto considerando-se a posse de casa própria $(p=0,002)$ como de automóvel $(p=0,016)$. Quanto ao grau de instrução, (fig. 12) a grande maioria dos indivíduos situou-se no nível médio ou primário, não havendo diferenças significativas entre os casos e os controles ( $\mathrm{p}=0,635)$. O inquérito referente aos hábitos alimentares mostrou: a) entre os que referiram consumo regular de alimentos sabidamente ricos em gorduras saturadas (fig. 13), observou-se prevalência significativamente maior entre os que consumiam embutidos ( $\mathrm{p}=0,013)$, não havendo diferença significativa com relação aos outros itens pesquisados, a saber: carne de porco $(p=0,566)$, bovina $(p=0,715)$, vísceras $(\mathrm{p}=0,154)$ e frituras $(\mathrm{p}=0,302)$; $\mathrm{b})$ houve associação significativa negativa entre a ocorrência de IAM e o consumo habitual de leite integral $(\mathrm{p}=0,027)$ mas não se observaram diferenças significativas no consumo de queijo integral $(\mathrm{p}=0,091)$, aves $(p=0,588)$, peixes $(p=0,372)$, vegetais verdes $(p=0,244) e$ frutas frescas $(\mathrm{p}=0,073)$ quando comparados os dois grupos (fig. 14); c) não houve diferenças significativas entre o consumo de óleo de oliva e outros óleos vegetais (fig. 15, p=0,435 e $0,885)$ mesmo dividindo-se os consumidores em duas faixas de consumo, o que também ocorreu quando se comparou o consumo de manteiga e margarina (fig. 16,p=0,077e 0,839); d) não houve diferença significativa entre casos e controles relativamente ao consumo habitual de chá ou café (fig. 17, $\mathrm{p}=0,946$ e 0,266 ). O nível sérico médio do colesterol total (fig. 18) foi significativamente maior entre os $\operatorname{casos}(\mathrm{p}=0,000)$.

\section{Discussão}

Idade e sexo - Ambas as variáveis foram adequadamente emparelhadas, conforme previa a metodologia. Considerando-se, no entanto, que o emparelhamento foi obviamente determinado pelos casos e não pelos controles, tem-se que a faixa etária prevalente para a ocorrência de IAM no estudo foi

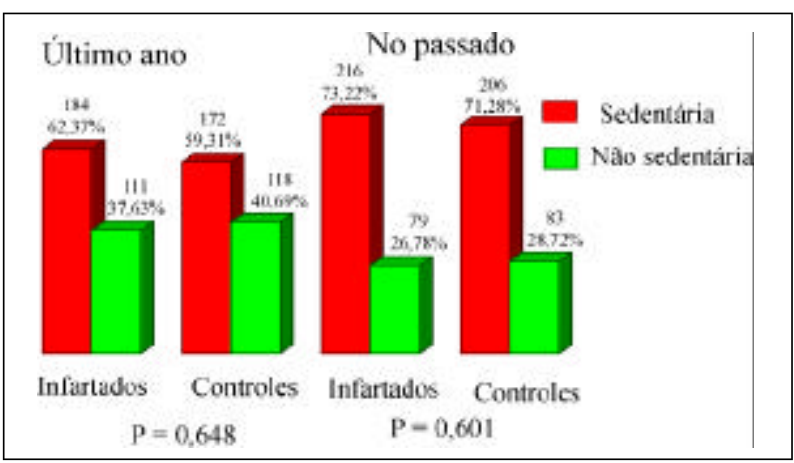

Fig. 9 - Atividade profissional II. *Não sedentária: atividade que obriga a caminhar mais da metade do tempo de trabalho.

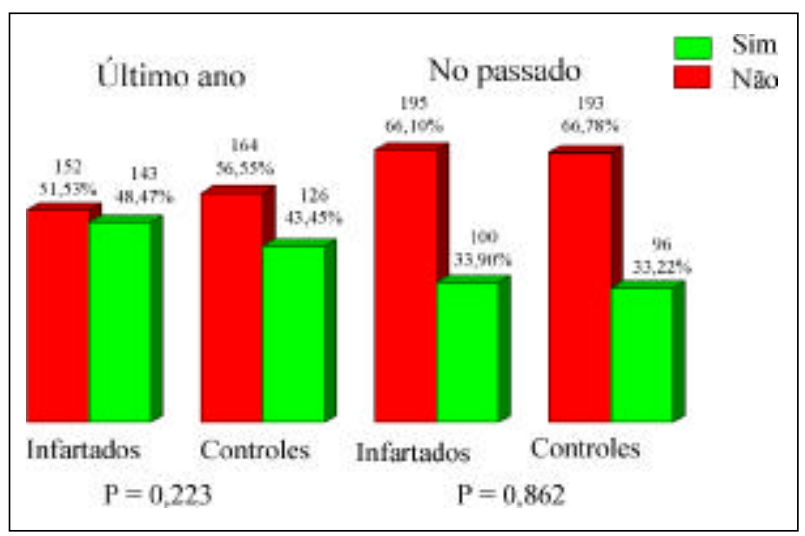

Fig. 10 - Atividade manual pesada.

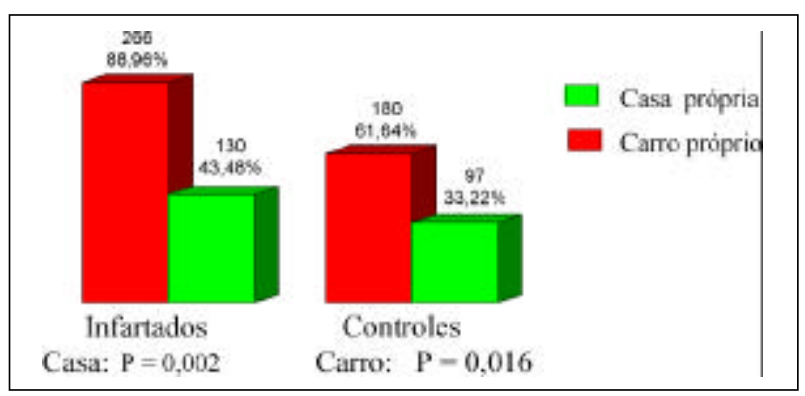

Fig. 11 - Condição socioeconômica. Relação entre a condição econômica e a ocorrência de IAM. Observe que o percentual dos que moram em casa própria e possuem automóvel é significativamente maior entre os infartados.

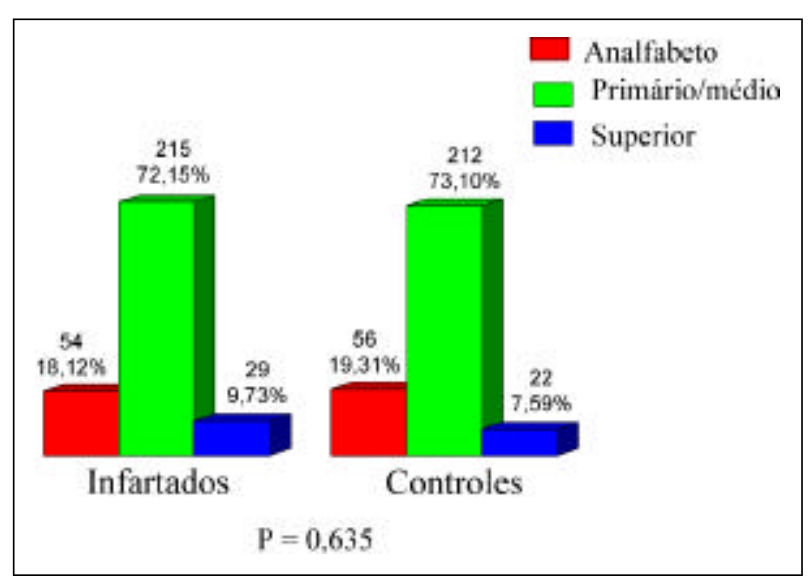

Fig. 12 - Grau de instrução. 


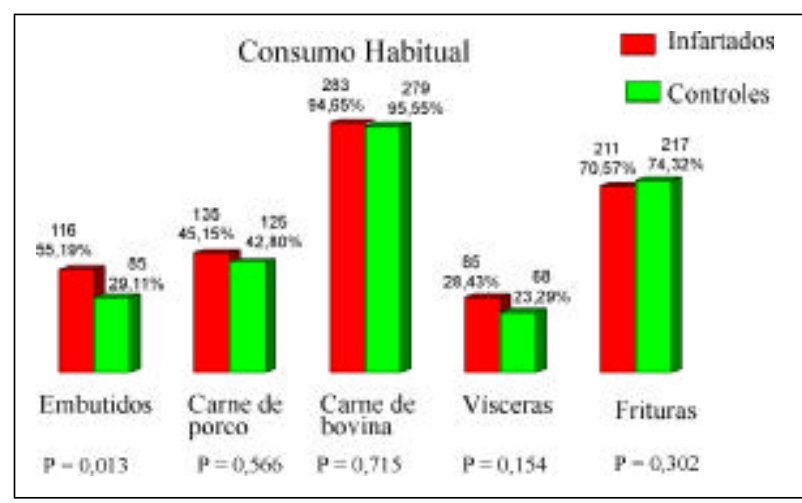

Fig. 13 - Hábitos alimentares Ia.

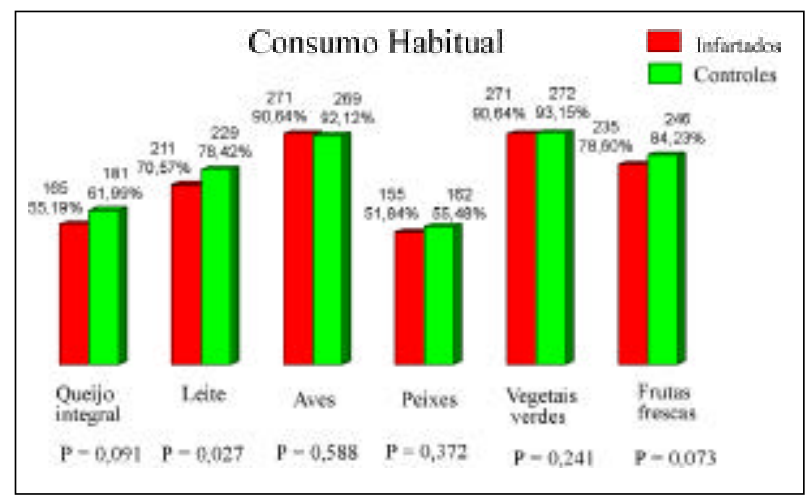

Fig. 14-Hábitos alimentares Ib.

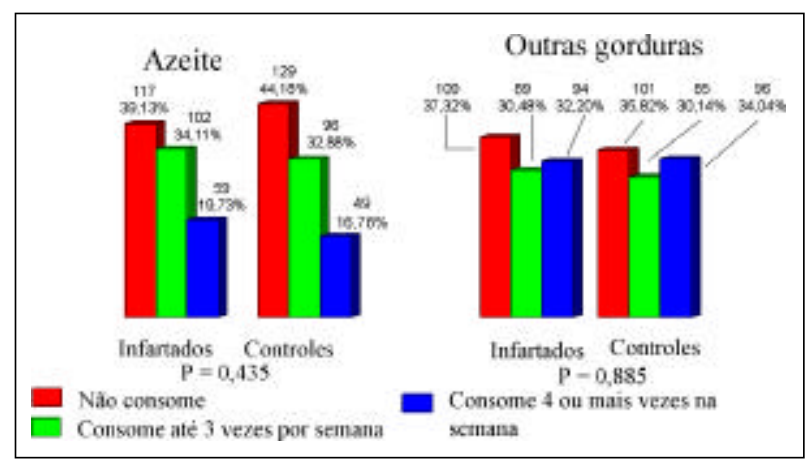

Fig. 15 - Hábitos alimentares II.

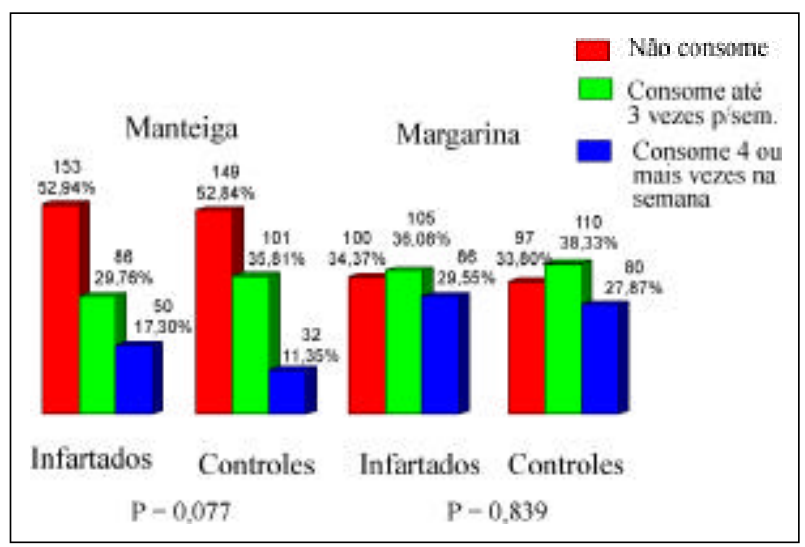

Fig. 16-Hábitos alimentares III.

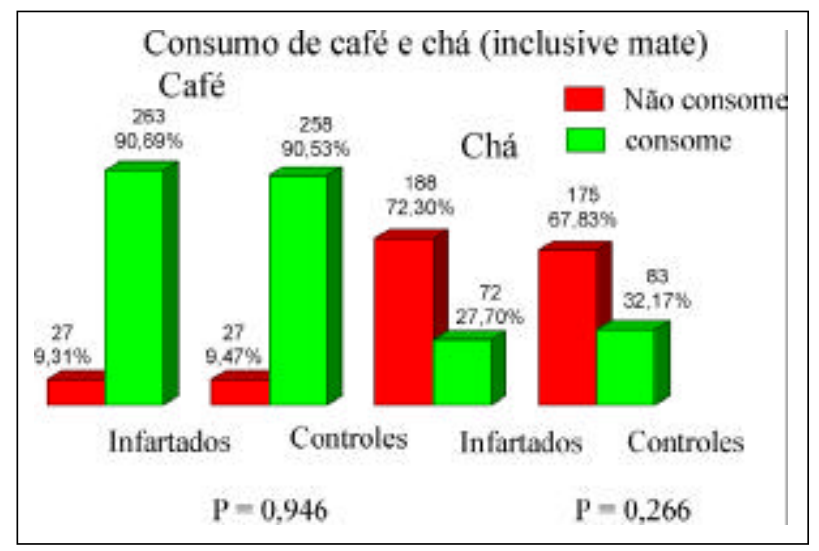

Fig. 17 - Hábitos alimentares IV.

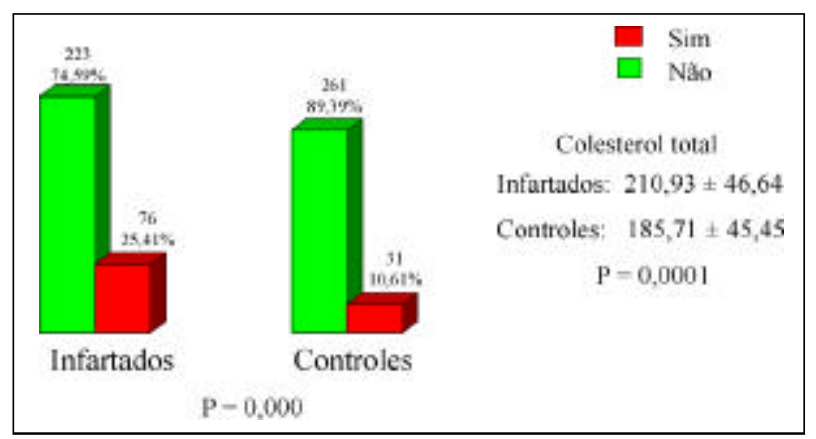

Fig. 18 - Associação com hipercolesterolemia.

de, em média, 58 anos. Faixa superior àquela apontada como sendo de maior risco para ocorrência de óbitos por doenças do aparelho circulatório no Brasil: a partir dos $45 \operatorname{anos}^{3}$, ou ainda menor ${ }^{6}$. Os resultados da Argentina neste mesmo estudos FRICAS também apontaram faixa etária mais alta: $57 \pm 36$ anos para os homens e 66,69 anos para as mulheres ${ }^{7}$. Uma explicação plausível para a prevalência em faixa etária mais tardia é a de que, no presente estudo, o que se investigou foi a prevalência do IAM e não a mortalidade, o que pode, de fato, ser mais prevalente a partir de faixas mais baixas.

No que se refere à diferença entre os gêneros, o presente estudo mostrou que o IAM foi quase três vezes mais prevalente entre os homens, quando comparado às mulheres. Tal resultado mostra uma diferença maior, em benefício das mulheres, do que quando se analisa a mortalidade. Neste caso, a mortalidade proporcional por DCV entre os homens foi, em 1989, de 39,30\%, contra 33,12\% entre as mulheres ${ }^{8}$. Duas explicações são possíveis: $1^{\circ}$ ) o estudo como já citado, não foi de mortalidade e sim da ocorrência de IAM e $2^{\circ}$ ), os percentuais apontados no trabalho englobam as DCVs em geral e não apenas a DAC. No estudo argentino ${ }^{7}$, a proporção encontrada foi de 2,87 homens para cada mulher, bastante próxima da verificada no presente estudo.

História familiar e estado civil - A presença de história de DAC nos genitores foi significativamente maior entre os infartados, quando comparados aos controles, quer se considere a mãe $(42,14 \%$ x $30,82 \%, \mathrm{p}=0,007)$ ou o pai $(42,14 \%$ x 
$33,22 \%, \mathrm{p}=0,025)$, o que está de acordo com o esperado. Em relação ao estado civil, não se observaram diferenças significativas entre os dois grupos.

Hipercolesterolemia e obesidade - Os níveis de colesterol total, embora não muito elevados em termos da média absoluta $(210,93 \pm 46,64 \mathrm{mg} / \mathrm{dl}$ nos infartados), foi significativamente maior em relação aos controles $(185,71 \pm 45,45 \mathrm{mg}$ / $\mathrm{dl}, \mathrm{p}=0,0001)$. Por razões de custo, não se efetuaram determinações fracionadas de colesterol e de triglicérides, impedindo avaliação mais abrangente dessas variáveis como fator de risco. O resultado obtido reforça, no entanto, a validade da recomendação de que, em termos de prevenção primária, procure-se manter os níveis do colesterol total em valores inferiores a $200 \mathrm{mg} / \mathrm{dl}$ ou menos ${ }^{9}$. No que tange ao papel da obesidade, embora não se tendo efetuado a determinação do índice de massa corporal (IMC), observou-se que o peso médio dos infartados $(72,50 \pm 26,89 \mathrm{~kg})$ foi significativamente $(\mathrm{p}=0,0271)$ superior ao dos controles $(69 \pm 12,26 \mathrm{~kg})$ para altura média semelhante $(166,56 \pm 7,81$ x 166,66 $\pm 8,47 \mathrm{~cm})$.

Hipertensão arterial - A associação com HAS foi de $53,4 \%$ entre os casos e de $20,8 \%$ entre os controles $(\mathrm{p}=0,000)$, configurando existir, entre os que apresentam IAM, uma prevalência de HAS mais de três vezes superior à da população adulta brasileira, estimada em $15 \%$, resultado que corrobora a importância da hipertensão como fator de risco para a ocorrência de DAC.

Tabagismo - Entre os infartados, $41,69 \%$ eram fumantes, contra $27,2 \%$ dos controles ( $\mathrm{p}=0,000$ ), corroborando, como em relação à HAS, a importância do tabagismo como fator de risco. Não se estratificou o risco com base no número de cigarros fumados por dia, nem no tempo de duração do hábito, informações valiosas e que não foram adequadamente pesquisadas. No estudo argentino ${ }^{7}$, analisaram-se em separado os que fumavam até 25 cigarros ao dia e os que fumavam mais de 25 . No primeiro subgrupo, não houve diferença significativa entre fumantes e não fumantes. No segundo, enquadravam-se $19,8 \%$ dos casos contra $6,1 \%$ dos controles $(\mathrm{p}<0,01)$. Como não se compararam as médias e como a faixa de uso foi muito ampla (de 1 a 25 cigarros/dia), a única conclusão possível é a de que o risco aumenta entre os que fumam acima de 25 cigarros ao dia, não se podendo, entretanto afirmar o inverso. Aspecto digno de nota, pelas implicações em termos de política de saúde pública, é a comparação entre não fumantes e ex-fumantes. Declínio imediato na freqüência cardíaca tem sido observado durante tentativas de abandono do tabagismo ${ }^{10,11}$. Por outro lado, sabese que a persistência no hábito de fumar, além do dano inicial, é fonte de contínuo agravo ao aparelho cardiovascular. No presente estudo, concordantemente com estas observações, não se observaram diferenças significativas entre fumantes e não fumantes $(\mathrm{p}=0,071)$ no que se refere ao risco para ocorrência de IAM. O achado embasa assim, a validade de se abandonar o hábito, em qualquer época.

Atividade física e profissional - A prática adequada e regular de atividade física tem sido admitida como uma das mais importantes e eficazes medidas na prevenção da DAC e na preservação da saúde e da qualidade de vida das pes- soas ${ }^{12,13}$.Em decorrência, estilo de vida sedentário tem sido considerado fator que aumenta o risco de se desenvolver aterosclerose coronária e se instalar quadro de IAM. Um estilo de vida sedentário inclui não somente a prática regular de atividade física, mas também, o tipo de atividade profissional. Ambos os aspectos foram analisados no presente trabalho, tendo sido verificado resultados que se afiguram contraditórios. A ocorrência de IAM foi significativamente menor entre os que cultivaram no passado, (há mais de 1 ano) o hábito de caminhar. Porém, não houve diferença significativa entre os que não caminhavam e os que o fizeram apenas no último ano. $\mathrm{O}$ achado sugere que o efeito protetor do hábito de caminhar não é imediato, manifestando-se apenas, no mínimo, após um ano de prática. Não foi constatada diferença significativa quanto à prática de esportes, tanto no presente, como no passado. Cabe aqui a possibilidade de não haver sido levada em conta, nas respostas, a pré-condição de que a prática de esportes fosse, pelo menos, por três vezes por semanae, no mínimo, por 45min de duração, comoé recomendado pela American College of Sports Medicine ${ }^{14}$.

Com relação à atividade profissional, não se observou, no presente estudo, diferença significativa entre os casos e controles, quanto ao tipo de atividade, se sedentária ou não e nem quanto ao fato de haver ou não atividade manual envolvida no tipo de ocupação. O achado conflita com os dados da literatura, visto que desde o início da década de 50, já se propunha a existência de correlação entre o tipo de trabalho e o risco de se desenvolver DAC ${ }^{15,16}$. Mais tarde, Haynes e Feinleib ${ }^{17}$ averiguaram os fatores de risco ocupacionais e comportamentais entre as mulheres participantes do estudo de Framinghan, tendo constatado maior prevalência de CAD entre as mulheres que trabalhavam, em comparação às donas de casa, considerando a faixa etária dos 45 ao 64 anos. No Bristh Regional Heart Study ${ }^{18}$, verificou-se que o risco de desenvolver DAC foi significativamente maior entre os trabalhadores manuais, muito embora essa diferença tenha sido atribuída à maior prevalência de tabagismo entre os que desenvolviam trabalho manual. Mesmo removendo-se este fator, no entanto, o excesso de risco, embora menor, permaneceu significativo ( $\mathrm{p}=0,08)$. A avaliação do peso da atividade profissional, como fator de risco para DAC, esbarra, justamente, na dificuldade de se isolarem os outros fatores envolvidos, que incluem não apenas a atividade física, mas também a condição socioeconômica e educacional e, principalmente, o grau e o tipo de estresse ocupacional. Como se sabe, o estresse ocupacional, resulta, principalmente, da conjunção de alto grau de responsabilidade com baixo poder de decisão. Resulta, também, da insatisfação profissional, ligada ao exercício de tarefas repetitivas e às quais o próprio trabalhador atribui pouca importância ${ }^{19}$. Este é o caso, justamente, da maioria dos que trabalham em atividades predominantemente manuais e não sedentárias. Esta pode ter sido a razão pela qual não se constataram, no presente estudo, diferenças relacionadas à atividade profissional entre os casos e os controles.

Nível educacional e condição socioeconômica - A associação entre um pobre estado de saúde e um baixo nível 
socioeconômico tem sido observada ao longo dos séculos. Com relação às DCVs, estudos prospectivos e um considerável corpo de evidências relativamente novas, têm apontado para a constatação de que uma baixa condição socioeconômica constitui-se em fator independente de risco ${ }^{20}$. Há, portanto, uma relação inversa, de sorte que, quanto mais alto o nível socioeconômico, mais baixa a ocorrência das DCVs. No que se refere especialmente ao subgrupo da DAC, havia, até há algumas décadas, a noção contrária, ou seja, a de que a relação seria direta. Em 1971, Cassel e col ${ }^{21}$, examinaram a associação entre DAC e nível socioeconômico no Evans County Georgia Heart Study e verificaram que, ao contrário do que se acreditava, a incidência de DAC ajustada para a idade, em sete anos de observação em homens brancos americanos, foi virtualmente a mesma, tanto entre os de alta com os de baixa condição socioeconômica. Posteriormente, o Whitehall Study ${ }^{22}$ e outros três importantes estudos epidemiológicos, realizados em Chicago ${ }^{23}$, mostraram uma nítida relação inversa entre a ocorrência de mortalidade por DAC e nível socioeconômico ${ }^{23}$. Na análise dos indicadores da condição socioeconômica, além da faixa salarial, sobressaí-se o nível educacional. Baseados nos dados fornecidos pela US National Longitudinal Mortality Study, Rogot e col ${ }^{24}$ mostraram haver forte relação inversa entre a mortalidade por todas as causas com a faixa de renda e nível educacional.

Os resultados do presente estudo, no entanto, não mostraram correlação entre o grau de instrução e a ocorrência de IAM, não havendo diferença significativa, quando comparados analfabetos com os de instrução primária, média ou superior. Também ao contrário da literatura, constatou-se, nesse estudo, haver uma relação direta entre a ocorrência de IAM e a condição econômica, visto haver sido verificada associação significativa entre o fato de se possuir casa e carro e a presença de DAC. É possível que, em nosso meio, a maior expectativa de vida e menor exposição a doenças ligadas às condições de moradia e de vida explique a maior prevalência de DAC, entre os mais favorecidos econômica e socialmente.

Hábitos alimentares - Embora tenha se verificado, no presente estudo, associação entre hipercolesterolemia e IAM e, mesmo, entre obesidade e IAM, não se observou influência significativa dos hábitos alimentares, com exceção do consumo habitual de embutidos, significativamente maior entre os que sofreram infarto. Vale destacar não haver sido observada diferença significativa, entre os dois grupos, com relação ao consumo de manteiga, comparado ao de margarina, e de café, comparado ao chá.

$\mathrm{O}$ presente estudo apresenta algumas limitações. Observa-se que a maior parte da casuística originou-se do estado de São Paulo, com larga predominância de um único centro (Marília), o que, sem dúvida, compromete a aceitação dos resultados como sendo representativos de todo o país. Esta constatação não altera, no entanto, a premissa que embasou o presente trabalho, ou seja, a de que a população brasileira, mesmo do estado de São Paulo, é diferente das amostras populacionais estudadas anteriormente, tanto do ponto de vista racial como socioeconômico. Do ponto de vista metodológico, alguns aspectos deixaram a desejar: não houve dosagem do triglicérides nem fracionamento do colesterol, não se aferiu o IMC e nem se estratificou o excesso de risco associado ao tabagismo, com base no número de cigarros fumados. Outras limitações relacionaram-se às características e desenho do estudo e ao tratamento estatístico. Tratou-se de um estudo caso-controle hospitalar que incluiu serviços predominantemente voltados para assistência cardiológica e que dispunham de unidade coronária. Este fato, se por um lado facilitou e conferiu credibilidade à seleção dos casos de infarto, dificultou a seleção dos controles. Destes últimos, procurou-se excluir portadores de doença ou condição capazes de gerar confusão entre as variáveis analisadas. A preocupação em emparelhar casos e controles por idade, sexo e centro de origem, diminuiu grande parte das possibilidades de confusão, mas não as eliminou por completo. No que se refere à análise estatística, os resultados apresentados foram estudados em abordagem univariadas, o que, sem dúvida, compromete o caráter conclusivo do trabalho. Tal fato não invalida, no entanto, o valor informativo, quanto ao papel das variáveis analisadas como fatores predisponentes e de risco, para a ocorrência do IAM no Brasil.

Aspecto importante diz respeito à confiabilidade das informações prestadas, ou seja, na aferição (medição) das variáveis incluídas no estudo. A possibilidade de erro advém, aqui, da impossibilidade de confirmar a veracidade da maioria das informações prestadas pelos pacientes, um vez que mais de um quarto (casos e controles) era constituída de analfabetos, proporção muito superior, provavelmente, àquela constante dos estudos realizados em países do primeiro mundo e mesmo na Argentina. Tentou-se reduzir ao mínimo o viés daí advindo, através da aplicação de um questionário simples e objetivo, com alternativas claramente apresentadas. Além disso, os investigadores do estudo foram orientados no sentido de dirimirem exaustivamente as dúvidas, por ocasião da coleta de dados.

Concluindo, os achados do presente estudo ratificaram o que já havia sido firmado em estudos epidemiológicos anteriores, tanto os internacionais, já citados ${ }^{1,2}$, como alguns estudos nacionais regionais ${ }^{25}$. Assim, tendo em vista as limitações mencionadas, os resultados obtidos permitiram observar: 1) HAS, hipercolesterolemia e o hábito de fumar, associaram-se fortemente à ocorrência de IAM; 2) houve associação significativa entre a ocorrência de IAM e a presença de DM e antecedentes familiares positivos para doença coronária; 3 ) a prevalência de IAM foi significativamente menor entre os que cultivavam, já há mais de um ano, o hábito de caminhar; 4) a ocorrência de IAM foi significativamente maior entre aqueles de maior peso corporal em relação à altura; 5) houve associação significativa entre a ocorrência de IAM e o consumo habitual de embutidos e, 6) diferentemente do que é relatado na literatura internacional, a ocorrência de IAM foi significativamente mais prevalente entre os de melhores condições socioeconômicas (carro e casa própria).

Investigadores do FRICAS (Brasil): Abrão Afiune (Goiânia, GO), AndréLabrounie(Marília, SP), Antonio Carlos Assunção (Araras/Leme, SP), Antonio Carlos Prestes (Vitó- 
ria/Vila Velha, ES), Aristóteles Conte Alencar(Manaus, AM), Hermes T. Xavier (Santos, SP), João Carlos Bosso (Presidente Prudente, SP), José Carlos Nicolau (São José do Rio Preto, SP), José Xavier de Melo Fo (São Luis, MA), Marco Aurélio
Barros (João Pessoa, PB), Nasser Sarkis Simão(Brasília, DF), Nabil Ghorayeb (São Paulo, SP), Pedro Pimentel (Porto Alegre, RS), Roberto Franken (São Paulo, SP), Roberto Verzola (São Carlos, SP) Takao Kawamura (Araçatuba, SP).

\section{Referências}

1. Vemura K, Piza Z - Trends in cardiovascular disease mortality in industrialized countries since 1950. Word Health Statist Quart 1988; 41: 155-78.

2. Davies MJ, Wolf N - Atherosclerosis: What is it and why does it accur? Br Heart J 1993; 69: 53-9.

3. Ministério da Saúde Fundação Nacional de Saúde Centro Nacional de Epidemiologia; Coordenação de Informática em Saúde Pública em Saúde. Estatísticas de mortalidade, 1988. Brasília, Centro de documentação, 1993.

4. Damber TR,Moore FE, Manm GV - Coronary heart disease in the Framinghan Study. Am J Public Health 1957; 47: 4-24.

5. Dawber TR - The Framinghan Study. The epidemiology of atherosclerotic disease. Haward University Press, 1980.

6. Chor D, Mendes da Fonseca MJ, Andrade CR - Doenças cardiovasculares. Comentários sobre a mortalidade precoce no Brasil. Arq Bras Cardiol, 64: 15-19.

7. Cruzzi M, Pozlosnik J, Pramparo $\mathrm{H}$ et al - Factores de riego para infarto agudo do miocardio en la Argentina. Rev Arq Cardiol 1996; 64(supp 11): 9-40.

8. Lotufo PA, Lolio CA - Tendência da mortalidade por doença isquêmica do coração no Estado de São Paulo: 1970 a 1989. Arq Bras Cardiol 1993; 61: 149-53.

9. $2^{\circ}$ Consenso Brasileiro Sobre Dislipidemias. Detecção - Avaliação - Tratamento. Arq Bras Cardiol 1996; 67(supl): 1-16.

10. Ward KD, Garvey AJ, Bliss RE - Evidence of transient heart rate change after smoking cessation. Psychopharmacology 1992; 106: 337-40.

11. Gidding SS, Xie X, Lin K et al - Função cardíaca em tabagistas e não-tabagistas: o estudo CARDIA. J Am Coll Cardiol (Ed Bras) 1995; 1: 378-81.

12. Mcginnis JM - The public health burden of a sedentary bifestyle. Med Sci Sports Exerc 1991; 24(6, suppl): S196-S200.

13. Paffenbarger RS, Hyde RT, Wing AL, Chung - Cheng H - Physical Activity, AllCause mortality and longevity of college alumini. N Engl J Med 1996; 314: 605-13.
14. American College of Sports Medicine Guidelines for Graded Exercise Testing and Exercise Prescription. Philadelphia: Lea and Feabiger, 1986.

15. Morris JN, Crawford MD - Coronary heart disease and physical activity of work. Evidence of a national necropsy survey. Br Med J 1953; 2: 1485-91.

16. Bruner D, Manelis G - Myocardial infarction among members of communal settlement in Israel. Lancet 1950; 3: 1049-57.

17. Haynes SG, Feinleb M - Women, work and coronary heart disease prospective findings from the Framinghan Heart Study. Am J Public Health, 1980; 70: 133-41.

18. Pocock SJ, Shaper AG, Cook DG, Phillips NA, Walker M - Social class diferences in ischaemic heart disease in British men. Lancet 1987; 2: 1987: 197-201.

19. Kristensen IS, Mancilha-Carvalho JJ - Ambiente, condições de trabalho e doenças cardiovasculares. Arq Bras Cardiol 1990; 55: 223-6.

20. Kaplan GA, Keil JE - Socioeconomic factor and cardiovascular disease: Rewiew of the literature. Circulation 1993; 88(part 1): 1973-88.

21. Cassel J, Heyden S, Bartel AG et al - Incidence of coronary heart disease by ethinic group, social class and sex. Arch Intern Med 1971; 128: 901-6.

22. Rose G, Marmot MG - Social class and coronary heart disease. Br Heart J 1981; 45: 13-19.

23. Lin K, Cedres LB, Stamler J et al - Relatioship of education to major risk factors and dealth from coronary heart disease, cardiovascular disease and all causes: findings of three Chicago epidemiologic studies. Circulation 1982; 66: $1308-14$.

24. Rogot, Sorlie PD, Johnson NJ, Schmitt A - Mortality study of 1.3 million persons by demographic, social and economic factors: 1979-1985 follow-up. National Institutes of Health, 1992 NIH publication $n^{\circ} 92$ - 3297: 1-5.

25. Achutti A, Achutti VR - Fatores de risco para aterosclerose. Elementos para descrição da situação no Rio Grande do Sul. Arq Bras Cardiol 1994; 63: 427-31. 